\title{
MRNA-based TriMix Melanoma Vaccine ECI-006
}

National Cancer Institute

\section{Source}

National Cancer Institute. mRNA-based TriMix Melanoma Vaccine ECI-006. NCI

Thesaurus. Code C154274.

A melanoma vaccine consisting of mRNAs encoding five different melanoma tumorassociated antigens (TAAs) and a TriMix platform comprised of three mRNAs encoding for constitutively activated toll-like receptor 4 (caT LR4), CD40 lig and (CD40L), and CD70, with potential immunomodulatory and antineoplastic activities. Upon intranodal injection, mRNA based TriMix vaccine $\mathrm{ECl}-006$ may stimulate the immune system to mount both humoral and cellular responses against tumor cells expressing the five TAAs specific to the vaccine, potentially decreasing cellular proliferation of cells expressing these antigens. The TriMix adjuvants CD4OL and caT LR4 promote the generation of mature and active dendritic cells (DCs), and CD70 provides a costimulatory signal to CD27+ naive T-cells, thereby supporting $\mathrm{T}$-cell proliferation and inhibiting $\mathrm{T}$-cell apoptosis. 